On the helix-coil transition in alanine based polypeptides in gas phase

Yanjie Wei, Walter Nadler, and Ulrich H. E. Hansmann

Citation: The Journal of Chemical Physics 126, 204307 (2007); doi: 10.1063/1.2734967

View online: https://doi.org/10.1063/1.2734967

View Table of Contents: http://aip.scitation.org/toc/jcp/126/20

Published by the American Institute of Physics

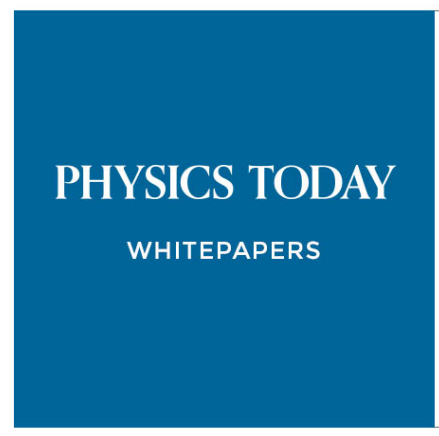
systems can do 


\title{
On the helix-coil transition in alanine based polypeptides in gas phase
}

\author{
Yanjie $\mathrm{Wei}^{\mathrm{a})}$ and Walter Nadler ${ }^{\mathrm{b})}$ \\ Department of Physics, Michigan Technological University, Houghton, Michigan 49931 \\ Ulrich H. E. Hansmann ${ }^{\text {c) }}$ \\ Department of Physics, Michigan Technological University, Houghton, Michigan 49931 and \\ John-von-Neumann Institute for Computing, Forschungszentrum Jülich, D-52425 Jülich, Germany
}

(Received 5 March 2007; accepted 3 April 2007; published online 24 May 2007)

\begin{abstract}
Using multicanonical simulations, the authors study the effect of charged end groups on helix formation in alanine based polypeptides. They confirm earlier reports that neutral polyalanine exhibits a pronounced helix-coil transition in gas phase simulations. Introducing a charged $\mathrm{Lys}^{+}$at the $\mathrm{C}$ terminal stabilizes the $\alpha$ helix and leads to a higher transition temperature. On the other hand, adding the $\mathrm{Lys}^{+}$at the $\mathrm{N}$ terminal inhibits helix formation. Instead, a more globular structure was found. These results are in agreement with recent experiments on alanine based polypeptides in gas phase. They indicate that present force fields describe accurately the intramolecular interactions in proteins. (C) 2007 American Institute of Physics. [DOI: 10.1063/1.2734967]
\end{abstract}

\section{INTRODUCTION}

First principles calculations of protein folding have remained a computationally hard problem. This is in part because the energetic and entropic balance of a solvated protein in equilibrium is complicated to express on a computer. Other problems arise from the inherent difficulties in sampling the rough energy landscape of a protein. It is not $a$ priori clear what contributes most to the numerical shortcomings: poor sampling techniques, lack of accuracy in the force fields that describe the intramolecular forces in a protein, or the crude approximations in the modeling of proteinsolvent interaction. The introduction of high-resolution ion mobility measurements, which allow experiments to be performed on biological molecules in gas phase, ${ }^{1,2}$ has opened a way to simplify the problem. By comparing simulations with gas-phase experiments, one can avoid the complications arising from the modeling of protein-solvent interactions. Hence, gas-phase experiments offer a way to test whether current force fields describe correctly the intramolecular forces and the ability of present simulation techniques to sample accurately low-energy configurations.

Such evaluation of methods and energy functions through comparison with gas phase experiments is done best with simple systems. Often used are homopolymers of amino acids. For instance, the helix-coil transition has been studied extensively with polyalanine. ${ }^{3-5}$ Numerical results indicate a strong propensity to form helices if the temperature is below a certain critical value. On the other hand, the experimental observations seemed to contradict those theoretical results. For this reason, it was conjectured in Ref. 6 that the strong helix-coil transition for polyalanine in gas-phase simulations is an artifact of the utilized energy function. However, the deviations may also result from differences in the systems

\footnotetext{
a)Electronic mail: yawei@mtu.edu

${ }^{b)}$ Electronic mail: wnadler@mtu.edu

${ }^{c)}$ Electronic mail: hansmann@mtu.edu, u.hansmann@fz-juelich.de
}

studied. Gas phase experiments require partially charged molecules while in simulations it is commonly assumed that the molecule has no total charge.

In order to settle this question and quantify the effects of charged end groups, we have simulated three alanine based polypeptides in gas phase. The first one is $\mathrm{Ala}_{10}$, which we are familiar with from earlier work. ${ }^{4-9}$ Simulations of this neutral molecule are supplemented by those of two charged polypeptides. In Ace-Lys ${ }^{+}-\mathrm{Ala}_{10}$ the charged group sits at the $\mathrm{N}$ terminal while in Ace- $\mathrm{Ala}_{10}$-Lys ${ }^{+}$the charge sits at the $\mathrm{C}$ terminal. Our results confirm the experimental findings that capping the $\mathrm{N}$ terminal by a positively charged lysine destabilizes the helix and leads to a more globular lowtemperature structure, while a $\mathrm{C}$ terminal $\mathrm{Lys}^{+}$can stabilize the $\alpha$ helix.

\section{METHODS}

Our simulations utilize the ECEPP/3 force field ${ }^{10}$ as implemented in the 2005 version of the program package SMMP. ${ }^{11,12}$ Here the interactions between the atoms within the homopolymer chain are approximated by a sum $E_{\mathrm{ECEPP} / 3}$ consisting of electrostatic energy $E_{C}$, a Lennard-Jones term $E_{\mathrm{LJ}}$, hydrogen-bonding term $E_{\mathrm{HB}}$, and a torsion energy $E_{\text {tor }}$ :

$$
\begin{aligned}
E_{\mathrm{ECEPP} / 3}= & E_{C}+E_{\mathrm{LJ}}+E_{\mathrm{HB}}+E_{\mathrm{tor}} \\
= & \sum_{(i, j)} \frac{332 q_{i} q_{j}}{\epsilon r_{i j}}+\sum_{(i, j)}\left(\frac{A_{i j}}{r_{i j}^{12}}-\frac{B_{i j}}{r_{i j}^{6}}\right) \\
& +\sum_{(i, j)}\left(\frac{C_{i j}}{r_{i j}^{12}}-\frac{D_{i j}}{r_{i j}^{10}}\right)+\sum_{l} U_{l}\left(1 \pm \cos \left(n_{l} \xi_{l}\right)\right),
\end{aligned}
$$

where $r_{i j}$ is the distance between the atoms $i$ and $j, \xi_{l}$ is the $l$ th torsion angle, and energies are measured in $\mathrm{kcal} / \mathrm{mol}$.

The above defined energy function leads to a landscape that is characterized by a multitude of minima separated by high barriers. In order to enhance sampling, we therefore utilize the multicanonical approach, ${ }^{13,14}$ as described in 


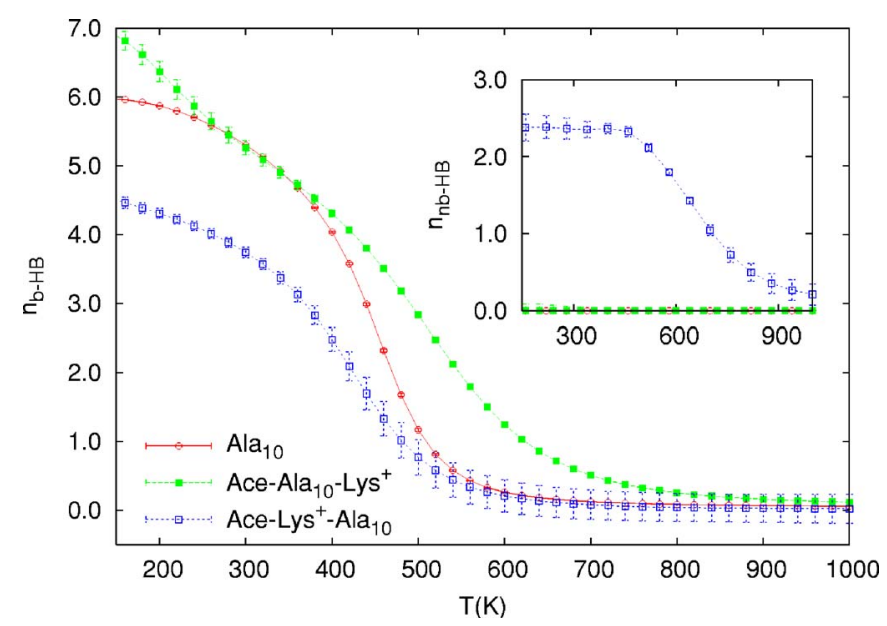

FIG. 1. (Color online) Average helicity, measured by the average number of backbone hydrogen bonds $\left\langle n_{\mathrm{b}-\mathrm{HB}}\right\rangle$, as function of temperature $T$ for the three polypeptides, obtained from a multicanonical simulation with $5 \times 10^{6}$ sweeps. $\mathrm{Ala}_{10}$ (open circles), Ace-Ala $10^{-} \mathrm{Lys}^{+}$(open squares), and Ace-Lys ${ }^{+}-\mathrm{Ala}_{10}$ (filled squares); error bars are included and are mostly about the symbol size or less. The inlay shows the nonbackbone hydrogen bonds $\left\langle n_{\mathrm{nb}-\mathrm{HB}}\right\rangle$, a measure of tertiary interactions.

Ref. 15. Configurations are weighted with a term $w_{M U}(E)$ determined iteratively ${ }^{16}$ such that the probability distribution obeys

$$
P_{M U}(E) \propto n(E) w_{M U}(E) \approx \mathrm{const},
$$

where $n(E)$ is the spectral density of the system. Thermodynamic averages of an observable $\langle O\rangle$ at temperature $T$ are obtained by reweighting: ${ }^{17}$

$$
\langle O\rangle(T)=\frac{\int d x O(x) e^{-E(x) / k_{B} T} / w_{M U}[E(x)]}{\int d x e^{-E(x) / k_{B} T} / w_{M U}[E(x)]},
$$

where $x$ counts the configurations of the system.

Various quantities are measured during simulations for further analysis. These include the energy $E$, the radius of gyration $R_{\mathrm{gy}}$ and the end-to-end distance $R_{\mathrm{ee}}$ as measures of the geometrical size, and the helicity. Here we define the helicity by the number of backbone hydrogen bonds $n_{\mathrm{b}-\mathrm{HB}}$ between residues $(i, i+4)$, which is characteristic for $\alpha$ helices. We also monitored the number of nonbackbone hydrogen bonds $n_{\mathrm{nb}-\mathrm{HB}}$, as a measure of tertiary interactions. Finally, we recorded the lowest energy configurations.

\section{RESULTS AND DISCUSSIONS}

As we are interested in the helix-coil transition in alanine based polypeptides, we start our investigation with an analysis of the average helicity for the three peptides, measured by the average number of backbone hydrogen bonds $\left\langle n_{\mathrm{b}-\mathrm{HB}}\right\rangle$. This quantity is displayed as a function of temperature in Fig. 1. For the neutral molecule, $\mathrm{Ala}_{10}$, a sharp transition is observed that separates a disordered high temperature phase with vanishing helicity from an ordered lowtemperature phase where most configurations have high helicity. Not surprisingly, the minimal energy configuration exhibits a fully formed $\alpha$ helix and is displayed in Fig. 2(a). Displaying the specific heat as a function of temperature in Fig. 3, we observe a sharp peak which indicates that this

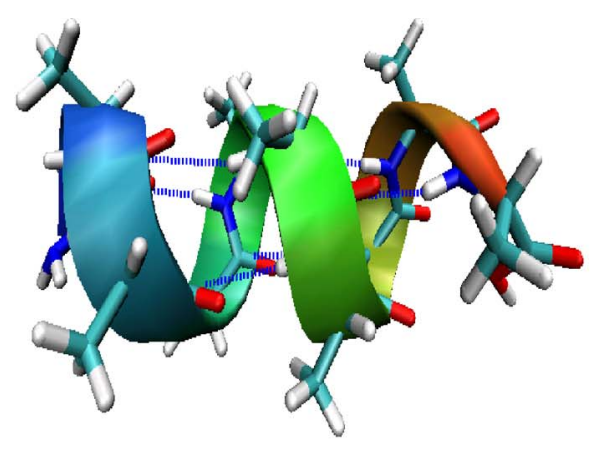

(a) $\mathrm{Ala}_{10}$

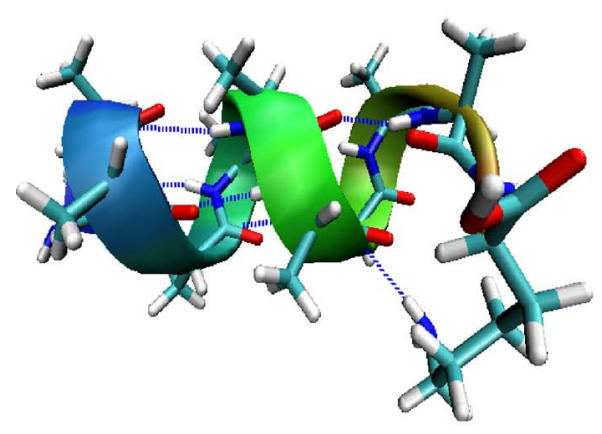

(b) Ace-Ala $0_{10}-\mathrm{Lys}^{+}$

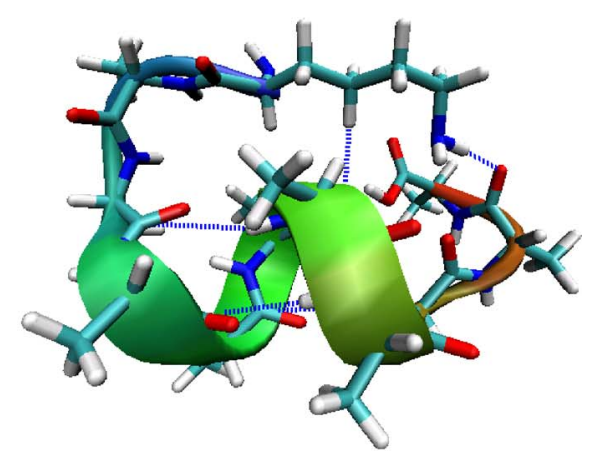

(c) Ace-Lys ${ }^{+}-\mathrm{Ala}_{10}$

FIG. 2. (Color online) Minimal energy configurations of (a) $\mathrm{Ala}_{10}$, (b) Ace-Ala $10-\mathrm{Lys}^{+}$, and (c) Ace-Lys ${ }^{+}-\mathrm{Ala}_{10}$, obtained by minimizing the lowest energy configuration found during the simulation. The dashed lines show hydrogen bonds. The pictures have been drawn with vMD (Ref. 20).

helix-coil transition takes place at a "critical" temperature $T_{C}=462 \mathrm{~K} \pm 5 \mathrm{~K}$. Note that this value is about $40 \mathrm{~K}$ higher than the corresponding temperature $427 \mathrm{~K} \pm 7 \mathrm{~K}$ for the older ECEPP/2 force field ${ }^{9}$ although the two transitions differ little otherwise (data not shown).

In order to study the effect of charges on the stability of an $\alpha$ helix, we perform a second simulation with Ace-Ala $10^{-} \mathrm{Lys}^{+}$. In this molecule, a positively charged Lys residue is added at the $\mathrm{C}$ terminal end of the chain. The measured values for the helicity of Ace- $\mathrm{Ala}_{10}-\mathrm{Lys}^{+}$are similar to $\mathrm{Ala}_{10}$, forming again a single extended helix upon lowering the temperature (see Fig. 1). Note that the peak in specific heat (Fig. 3) is with $T_{C}=557 \pm 19 \mathrm{~K}$ at an $\approx 100 \mathrm{~K}$ higher temperature and is much wider. This increase in the transition temperature indicates that the charged $\mathrm{Lys}^{+}$at the $\mathrm{C}$ terminal favors the $\alpha$ helix through stabilizing the helix 


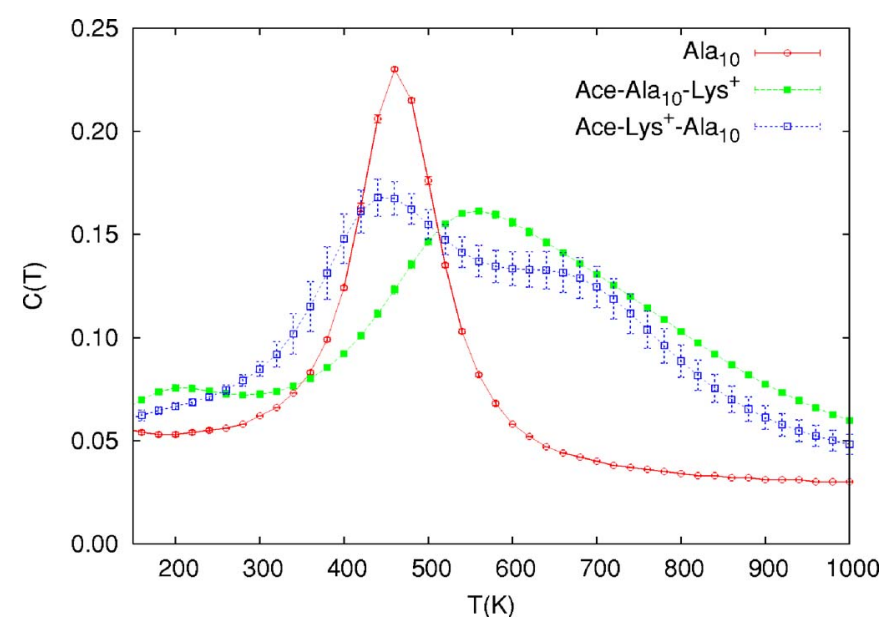

FIG. 3. (Color online) Specific heat $C(T)$ as function of temperature $T$ for the three polypeptides, obtained from a multicanonical simulation with 5 $\times 10^{6}$ sweeps. Symbols as in Fig. 1. Error bars are included and are mostly about the symbol size or less.

dipole. Parts of the $\alpha$ helix survive even up to higher temperatures, widening the transition regime considerably. The stable $\alpha$ helix structure is also seen in the minimal energy configuration displayed in Fig. 2(b). Note the additional hydrogen bond formed by the charged end group. Our results are consistent with recent experiments that also indicate stabilization by charged groups capping the $\mathrm{C}$ terminus and by the interaction of the charge with the helix dipole. ${ }^{18}$

The third peptide that we have studied is Ace-Lys ${ }^{+}-\mathrm{Ala}_{10}$. Here the charged Lys is added at the $\mathrm{N}$ terminal. For this peptide one finds only a small peak in the specific heat (see Fig. 3), just below that of $\mathrm{Ala}_{10}\left(T_{C}\right.$ $=449 \pm 37 \mathrm{~K}$ ), and a shoulder at higher temperatures. At low temperatures, its helicity (displayed in Fig. 1) is substantially lower than for the previous two peptides. Figure 2(c) displays the minimal energy configuration. Only a small partial helix is seen in the central region, while the end groups coil and form a more compact globular structure. The inlay in Fig. 1 indicates that nonhelical hydrogen bonds, stabilizing the globular structure, are formed already around $600 \mathrm{~K}$, above the small peak in specific heat. This formation of the globular structure as well as the joining up of the end groups are corroborated by the collapse of the molecule in that temperature range, as seen in the radius of gyration $\left\langle R_{\mathrm{gy}}\right\rangle$ as well as in the end-to-end distance $\left\langle R_{\text {ee }}\right\rangle$ in Fig. 4. For comparison, in the uncharged molecule the drop in $\left\langle R_{\mathrm{gy}}\right\rangle$ occurs at lower temperatures, i.e., at the helix-coil transition, with practically no change in the end-to-end distance, Ace-Ala 10 -Lys ${ }^{+}$, the charged helix-forming variant, shows a similar behavior. In contrast, the formation of the helical subsegment in Ace-Lys ${ }^{+}-\mathrm{Ala}_{10}$ does not show up significantly in the geometrical measures of that molecule. Hence, our data indicate that there is no well-defined helix-coil phase transition for Ace-Lys ${ }^{+}-\mathrm{Ala}_{10}$. Instead, the shoulder in the specific heat rather marks the collapse of the molecule, while the peak at lower temperatures marks the formation of the helical subsegment. The reason for the suppressed and only partial helix formation is the unfavorable interaction between the $\mathrm{N}$-terminal $\mathrm{Lys}^{+}$and the helix dipole, destabilizing the ex-

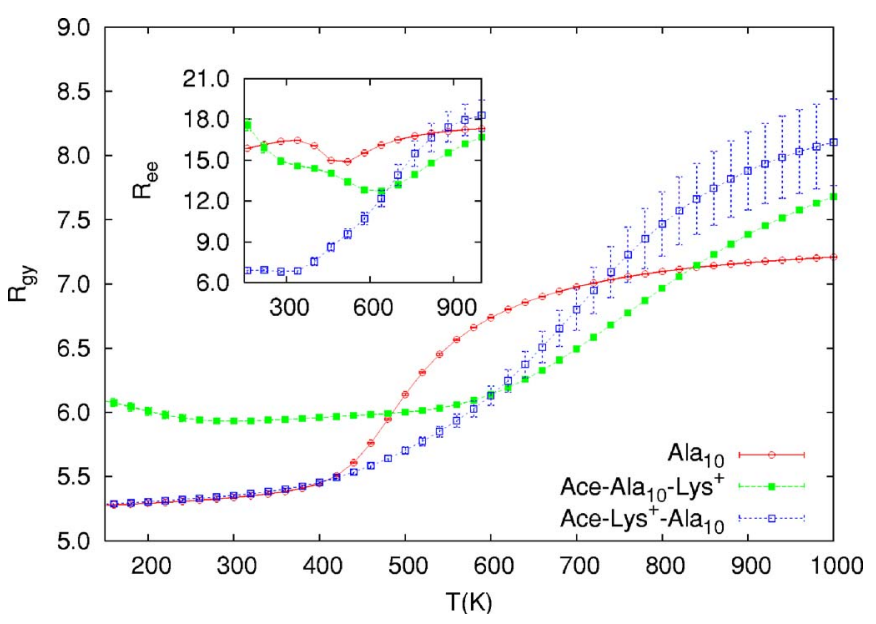

FIG. 4. (Color online) Average radius of gyration $\left\langle R_{\mathrm{gy}}\right\rangle$ as function of temperature $T$ for the three polypeptides, obtained from a multicanonical simulation with $5 \times 10^{6}$ sweeps. Symbols as in Fig. 1. Error bars are included and are mostly about the symbol size or less. The inlay shows the average end-to-end distance, $\left\langle R_{\mathrm{ee}}\right\rangle$.

tended helix. This conjecture is again supported by experimental finding in gas phase where the relative cross sections of this peptide also indicate configurations that are more compact than helices. ${ }^{6,21}$

\section{CONCLUSION}

This study has been motivated by a discrepancy between gas-phase experiments and first principles calculations of polyalanine that either could indicate shortcomings of our energy function, the ECEPP force field, or differences between the systems studied. As the original work in Ref. 9 focused on neutral molecules, while the experiments studied charged molecules, ${ }^{6}$ we have now compared simulations of polyalanine with either no charge, the charge at the $\mathrm{C}$ terminus, or at the $\mathrm{N}$ terminus. Our simulation for the charged molecules is now in agreement with the experimental results. Our results underline two points. First, when comparing gas phase experiments with simulations one has to make sure that the experimental settings are adequately described in the computer experiment. Second, the agreement between gas phase experiment and simulation suggests that the current generation of force fields describes the intramolecular forces within a protein sufficiently accurate to allow for correct secondary structure formation. The often observed failure of protein simulations in finding the correct structure (see, e.g., Ref. 19) therefore results likely from insufficient sampling and poor representations of the protein-solvent interaction.

\section{ACKNOWLEDGMENT}

Support by a research grant (CHE-0313618) of the National Science Foundation is acknowledged. 
${ }^{1}$ P. Dugourd, R. R. Hudgins, D. E. Clemmer, and M. F. Jarrold, Rev. Sci. Instrum. 68, 1122 (1997).

${ }^{2}$ R. R. Hudgins, J. Woenckhaus, and M. F. Jarrold, Int. J. Mass Spectrom. Ion Process. 165/166, 497 (1997).

${ }^{3}$ M. Kohtani, J. E. Schneider, T. C. Jones, and M. F. Jarrold, J. Am. Chem. Soc. 126, 16981 (2004).

${ }^{4}$ Y. Peng, U. H. E. Hansmann, and N. A. Alves, J. Chem. Phys. 118, 2374 (2003).

${ }^{5}$ M. Kohtani and M. F. Jarrold, J. Am. Chem. Soc. 126, 8454 (2004).

${ }^{6}$ B. S. Kinnear, D. T. Kaleta, M. Kohtani, R. R. Hudgins, and M. F. Jarrold, J. Am. Chem. Soc. 122, 9243 (2000).

${ }^{7}$ Y. Peng and U. H. E. Hansmann, Biophys. J. 82, 3269 (2002).

${ }^{8}$ N. A. Alves and U. H. E. Hansmann, Physica A 292, 509 (2001).

${ }^{9}$ U. H. E. Hansmann and Y. Okamoto, J. Chem. Phys. 110, 1267 (1999).

${ }^{10}$ M. J. Sippl, G. Némethy, and H. A. Scheraga, J. Phys. Chem. 88, 6231 (1984) and references therein.
${ }^{11}$ F. Eisenmenger, U. H. E. Hansmann, Sh. Hayryan, and C.-K. Hu, Comput. Phys. Commun. 138, 192 (2001).

${ }^{12}$ F. Eisenmenger, U. H. E. Hansmann, Sh. Hayryan, and C.-K. Hu, Comput. Phys. Commun. 174, 422 (2006).

${ }^{13}$ B. A. Berg and T. Neuhaus, Phys. Lett. B 267, 249 (1991).

${ }^{14}$ B. A. Berg and T. Celik, Phys. Rev. Lett. 69, 2292 (1992).

${ }^{15}$ U. H. E. Hansmann and Y. Okamoto, J. Comput. Chem. 14, 1333 (1993).

${ }^{16}$ B. A. Berg, J. Stat. Phys. 82, 323 (1996).

${ }^{17}$ A. M. Ferrenberg and R. H. Swendsen, Phys. Rev. Lett. 61, 2635 (1988); 63, 1658(E) (1989) and references given in the erratum.

${ }^{18}$ M. Kohtani, T. C. Jones, J. E. Schneider, and M. F. Jarrold, J. Am. Chem. Soc. 126, 7420 (2004).

${ }^{19}$ U. H. E. Hansmann, J. Chem. Phys. 120, 417 (2004).

${ }^{20} \mathrm{http} / / / \mathrm{www} . k s$.uiuc.edu/Research/vmd/

${ }^{21}$ R. R. Hudgins and M. F. Jarrold, J. Am. Chem. Soc. 121, 3494 (1999). 